\title{
Age-related obesity and type 2 diabetes dysregulate neuronal associated genes and proteins in humans
}

\author{
Mehran Rahimi i, ${ }^{1,}$, Manlio Vinciguerra ${ }^{2,3, *}$, Mojtaba Daghighi ${ }^{4}$, Behiye Özcan ${ }^{5}$, \\ Vishtaseb Akbarkhanzadeh ${ }^{6}$, Fareeba Sheedfar ${ }^{7}$, Marzyeh Amini ${ }^{8}$, Tommaso \\ Mazza ${ }^{9}$, Valerio Pazienza ${ }^{3}$, Mahdi M. Motazacker ${ }^{10}$, Morteza Mahmoudi ${ }^{11,12}$, Felix W. M. \\ De Rooij ${ }^{13}$, Eric Sijbrands ${ }^{13}$, Maikel P. Peppelenbosch ${ }^{14}$, Farhad Rezaee ${ }^{14,15}$ \\ ${ }^{1}$ Faculty of Medical Science, University Medical Center Groningen, University of Groningen, Groningen, The Netherlands \\ ${ }^{2}$ Institute for Liver and Digestive Health, Division of Medicine, University College London (UCL), London, UK \\ ${ }^{3}$ Gastroenterology Unit, IRCCS Casa Sollievo della Sofferenza, San Giovanni Rotondo, Italy \\ ${ }^{4}$ Department of Biomedical Engineering, University Medical Center Groningen, University of Groningen, Groningen, The Netherlands \\ ${ }^{5}$ Department of Endocrinology, Erasmus Medical Center, Rotterdam, The Netherlands \\ ${ }^{6}$ Institute Center-45, Medical Center, University of Amsterdam, The Netherlands \\ ${ }^{7}$ Department of Physiology, Radboud University Medical Center, Nijmegen, The Netherlands \\ ${ }^{8}$ Department of Epidemiology, University Medical Center Groningen, University of Groningen, Groningen, The Netherlands \\ ${ }^{9}$ Bioinformatics Unit, IRCCS Casa Sollievo della Sofferenza, San Giovanni Rotondo, Italy \\ ${ }^{10}$ Department of Clinical Genetics, Academic Medical Center, Amsterdam, The Netherlands \\ ${ }^{11}$ Department of Pediatrics, Stanford University School of Medicine, Stanford, CA, United States \\ ${ }^{12}$ Department of Nanotechnology and Nanotechnology Research Center, Faculty of Pharmacy, Tehran University of Medical \\ Sciences, Tehran, Iran \\ ${ }^{13}$ Department of cardiovascular genetics, Metabolism, Erasmus Medical Center, Rotterdam, The Netherlands \\ ${ }^{14}$ Department of Gastroenterology and Hepatology, Erasmus University Medical Center, University of Rotterdam, Rotterdam, \\ The Netherlands \\ ${ }^{15}$ Department of Cell Biology, University Medical Center Groningen, University of Groningen, Groningen, The Netherlands \\ *These authors have contributed equally to this work \\ Correspondence to: \\ Farhad Rezaee, e-mail: F.rezaee@erasmusmc.nl, F.Rezaee@med.umcg.nl \\ Keywords: aging, obesity, diabetes, age-related diabetes neuropathy, pancreas \\ Received: July 12, $2015 \quad$ Accepted: August 07, $2015 \quad$ Published: August 18, 2015
}

\section{ABSTRACT}

Despite numerous developed drugs based on glucose metabolism interventions for treatment of age-related diseases such as diabetes neuropathies (DNs), DNs are still increasing in patients with type 1 or type 2 diabetes (T1D, T2D). We aimed to identify novel candidates in adipose tissue (AT) and pancreas with T2D for targeting to develop new drugs for DNs therapy.

AT-T2D displayed 15 (e.g. SYT4 up-regulated and VGF down-regulated) and pancreas-T2D showed 10 (e.g. BAG3 up-regulated, VAV3 and APOA1 down-regulated) highly differentially expressed genes with neuronal functions as compared to control tissues. ELISA was blindly performed to measure proteins of 5 most differentially expressed genes in 41 human subjects. SYT4 protein was upregulated, VAV3 and APOA1 were down-regulated, and BAG3 remained unchanged in 1- Obese and 2- Obese-T2D without insulin, VGF protein was higher in these two groups as well as in group 3- ObeseT2D receiving insulin than 4-lean subjects. Interaction networks analysis of these 5 genes showed several metabolic pathways (e.g. lipid metabolism and insulin signaling).

Pancreas is a novel site for APOA1 synthesis. VGF is synthesized in AT and could be considered as good diagnostic, and even prognostic, marker for ageinduced diseases obesity and T2D. This study provides new targets for rational drugs development for the therapy of age-related DNs. 


\section{INTRODUCTION}

The homeostasis of blood glucose levels is maintained by both insulin and glucagon synthesis, which is functionally tightly regulated by the pancreatic $\beta$-cells and $\alpha$-cells respectively. When blood glucose levels rise (hyperglycemia), pancreatic $\beta$-cells synthesize and secrete insulin to the bloodstream to regulate glucose levels. On the contrary, when circulating glucose levels fall below normal values (hypoglycemia), pancreatic $\alpha$-cells produce and release glucagon to the bloodstream to tune up glucose concentrations via conversion of stored glycogen into glucose and its release into the circulation $[1,2]$. Thus, a disruption in blood glucose regulation leads to the hyperglycemia state so-called diabetes. There are two types of aged-related [3, 4] diabetes; type 1 diabetes (T1D) and type 2 diabetes (T2D). Diminished or dysregulation of insulin by $\beta$-cells in T1D and T2D respectively are caused by cell aging process. T1D is an autoimmune disease that pancreatic $\beta$-cells are targeted to be broken down by antibodies produced by immune B cells. In this regard, the main production site of insulin is diminished by $\beta$-cells [5-9]. In contrast, in age-related-T2D the pancreatic $\beta$-cells are still active and do synthesize insulin but at dysregulated levels and not sensitive enough [10-13]. In a normal state, there is a homeostatic balance and cross talk between skeletal muscle, liver and adipose tissue (AT) organs with respect to glucose levels, which is strictly under control of insulin [14]. In this regard, insulin 1- promotes glucose uptake and inhibits lipolysis by the skeletal muscle, 2stimulates glycogenesis and suppress glucose formation and release from the liver, and 3- stimulates adipogenesis and prevent lipolysis in AT-associated adipocytes [14]. In an obese state, insulin shows a function completely opposite to the normal state in these three organs. Moreover, it has been recently shown that other cells such as adipocytes, macrophages also synthesize insulin [15]. Patients with either T1D or T2D diabetes are at high risk for serious complications such as cardiovascular diseases (CVDs) [16] and diabetic neuropathy (DN) [17-23]. Obesity plays an important role in the development of T2D and its complications via the promotion of an inflammatory state [16]. In this regard, Systems biology could be a solution to find etiology of aged-related diseases such as T1D, T2D, neurodegenerative and cardiovascular diseases [24].

DNs are the most common micro-vascular complications that occur in both $\mathrm{T} 1$ and $\mathrm{T} 2$ diabetes (40-60\%) [18-20, 25, 26]. DNs-associated syndromes are classified into two groups; diffuse and focal. The former neuropathies are usually chronic and more common among diabetic patients, while focal neuropathies are acute [25]. In general, DNs occur in progressive damages of neuronal cells that commonly appear with symptoms such as severe pain, loss of sensation and disability [19, 22, 25].

Despite the general agreement that hyperglycemia plays a key role in initiation and development of DNs, the underlying mechanisms for the development of DNs is unknown and remains to be investigated [19, 22, 27]. Numerous studies have shown that hyperglycemia leads to the overload of electron transport chain in mitochondria and subsequent oxidative stress. The imbalance in the mitochondrial redox state, in favor of oxidation conditions, leads to the accumulation of reactive oxygen species (ROS). Subsequently, oxidative stress affects several metabolic pathways [such as insulin signaling pathway and protein kinase $\mathrm{C}(\mathrm{PKC})$ pathway; advanced glycation end products (AGEs) and mitogen activated protein kinase (MAPKs)] associated with glucose metabolism with enhanced oxidative stress and, in turn, inflammatory reactions [20, 21, 25, 28-32].

Although numerous drugs and therapies have been proposed to treat the DNs, the majority of these drugs and treatments were restricted to target the redox and glucose metabolic pathways $[20,32]$. None of the drugs that targeted glucose metabolic pathways showed any efficient effect in the treatment of DNs $[19,22]$. Also, despite identification of the genes [19] and metabolic pathways associated with DNs, the molecular causes of DNs events still occur in a large scale. These studies suggest that beside hyperglycemia other genetic components and factors may contribute to the development of DNs. For instance, tight links between oxidative stress and obesity were recently reported, which may provide new insight for investigation of the genes associated with DNs [21]. Meijer et al. have recently shown that metabolic dysfunction leads to the adipocytes hypertrophy (the main cause of obesity), which primes inflammation in AT $[15,16]$ and, in turn, is implicated in pathophysiological states such as energy storage disruption within adipocytes (e.g. triglycerides breakdown, FFAs, glycerol et cetera), insulin resistance (IR), and hyperglycemia as hallmark of T2D.

Feldman et al. reported several pathways involved in lipid, carbohydrate and energy metabolism modulating gene expression patterns in peripheral nerves on BKS db/ $\mathrm{db}$ mouse sciatic nerve. Although this study support the hypothesis that hyperglycemia play an important role in nerve damage, lipid metabolism may involve neuronal damage and development of DNs as well [19]. Since both pancreas and AT are key tissues involved in age-induced $\mathrm{T} 2 \mathrm{D}$ and its complications, this study was designed to investigate whether $\mathrm{T} 2 \mathrm{D}$ alters the expression of genes with a function in neuronal processes in human pancreas and AT and that might be implicated in the insurgence of DNs.

\section{RESULTS}

\section{The effect of type 2 diabetes on genes with a neuronal function}

\section{Pancreas}

To investigate whether there are genes with a neuronal function that could show highly and clear changes in expression and that caused by age-induced 
T2D, we measured mRNA expression in human pancreas and adipose tissue (AT) with T2D. As depicted in Table 1 and Figure 1, two genes $O L I G 1$ and $B A G 3$ were highly up-regulated and eight genes PRDM16, GLDNA, APOA1, $C C K B R, E D N 3, V A V 3, C X C R 4$, and GHRL were down regulated in pancreas with T2D as compared to pancreas control. Based on Gene Ontology database search, OLIG1 gene shows functions in neuron fate commitment, neurogenesis, generation of neurons, and neuron differentiation as shown in Figure 2. Based on database search, $B A G 3$ gene showed only a neural function (i.e. neuron part). There were 24 neuron functions found for these 10 genes in pancreas of which chemokine $\mathrm{C}-\mathrm{X}-\mathrm{C}$ receptor ( $\mathrm{CXCR} 4$ ) gene was shown to have a role in 17 neural functions based on Gene Ontology database (Figure 2). Among eight down-regulated genes in pancreas with T2D, VAV3 gene was highest down-regulated gene with approximately 246-fold (Table 1 and Figure 1).

\section{Adipose tissue (AT)}

The expression of 15 genes were found to be highly altered in AT caused by age-induced T2D (Table 2 and Figure 3). From these 15 genes, 8 gens were highly upregulated and 7 genes highly down-regulated (Table 2, and Figure 3). The expression of synaptotagmin-4 (SYT4) gene were tremendously high up-regulated (approximately 392-fold) in AT-T2D as compared to AT-control, while $V G F$ (neurosecretory protein) is highly down-regulated (approximately 119-fold) Table 2 and Figure 3). Another gene, which is very highly up-regulated in AT-T2D as compared to AT-control was MYRF (Myelin regulatory factor) as displayed in Table 2 and Figure 3. The fold changes of all 15 genes were reported in Table 2. Based on
Gene Ontology database search, 52 neural functions were found for these 15 genes (Figure 4). Although $V G F$ gene was highly down-regulated, only one neural function (i.e. neuropeptide hormone activity) was shown to be linked with this gene (Figure 4). SYT4 gene showed a link with 5 neural functions; 1- neurological system process, 2neurotransmitter secretion, 3- neurotransmitter transport 4- neuron part and 5- neuron projection. Based on Gene Ontology search, PTN and LRNN4 genes showed only link with one neural function (Figure 4) and both genes were up-regulated, while the other genes showed multiple neural functions.

\section{Pathway analysis}

The in-silico functional analysis conducted on the list of 25 genes, led to the identification of several molecule-function/disease connections, as shown in Figure 5. Focusing on SYT4, VGF, BAG3, APOA1, and $V A V 3$ and on their tightly related interactors, which in turn participated to lipid metabolism, insulin, protein kinase $\mathrm{C}$ (PKC), advanced glycation end (AGE) products and MAPK signaling pathways, as well as to the electron transport chain, oxidative stress and glucose metabolism biological processes, we have drawn a global interaction network (Figure 6). It contained 198 (13.8\%) multiprocess genes, with 31 (AKT2, CALM1, CALM2, CALM3, FOXA2, G6PC, HRAS, IKBKB, INSR, IRS1, MAPK1, MAPK3, MAPK8, MLYCD, PDK4, PRKAA1, PRKACA, PRKACB, PRKACG, PRKCG, PRKCZ, RAF1, SHC1, G6PD, PLA2G4A, SIRT1, SOD1, TNF, CASP3, EGFR and $M A P K 14$ ) genes participating to 3 processes, 7 (FOXO1, INS, MAP2K1, MAPK9, PRKCA, PRKCB and NFKB1) genes participating to 4 processes and 2 (AKT1 and

\section{Table 1: The list of highly up-regulated and down-regulated genes with a neuronal function detected in human control pancreas and pancreas with type 2 diabetes (T2D) using mRNA expression.}

\begin{tabular}{|c|c|c|c|c|c|}
\hline Protein ID & Protein description & pI & Mw (Da) & Gene name & $\begin{array}{l}\text { Pan-T2D vs Pan- } \\
\text { Cont (fold change) }\end{array}$ \\
\hline Q8TAK6 & Oligodendrocyte transcription factor 1 & 9.71 & 27,905 & OLIG1 & 38.0 \\
\hline O95817 & BAG family molecular chaperone regulator 3 & 6.46 & 61,595 & BAG3 & 34.2 \\
\hline Q9UKW4 & Guanine nucleotide exchange factor VAV3 & 6.64 & 97,776 & VAV3 & -246.8 \\
\hline P32239 & Gastrin/cholecystokinin type B receptor & 10.03 & 48,419 & CCKBR & -89.7 \\
\hline Q9HAZ2 & PR domain zinc finger protein 16 & 5.81 & 140,251 & PRDM16 & $-81,5$ \\
\hline P02647 & Apolipoprotein A1 & 5.56 & 30,778 & APOA1 & -65.7 \\
\hline P14138 & Endothelin-3 & 6.24 & 25,454 & EDN3 & -55.0 \\
\hline Q6ZMI3 & Gliomedin & 8.10 & 58,957 & GLDN & -43.0 \\
\hline Q9UBU3 & Appetite-regulating hormone & 5.35 & 12,911 & GHRL & -37.0 \\
\hline P61073 & C-X-C chemokine receptor type 4 & 8.46 & 39,746 & CXCR4 & -32.7 \\
\hline
\end{tabular}

Protein accession number, protein description, pI (isoelectric point), the Molecular weight (Mw), gene name of that protein, and fold change (Pancreas-T2D vs Pancreas-Control) are presented for each gene in this Table. The Mw expressed in Da. pI resulted from whole amino acid sequence of each protein and is derived from www.expasy.org. 


\section{pancreas-T2D vs pancreas-cont}

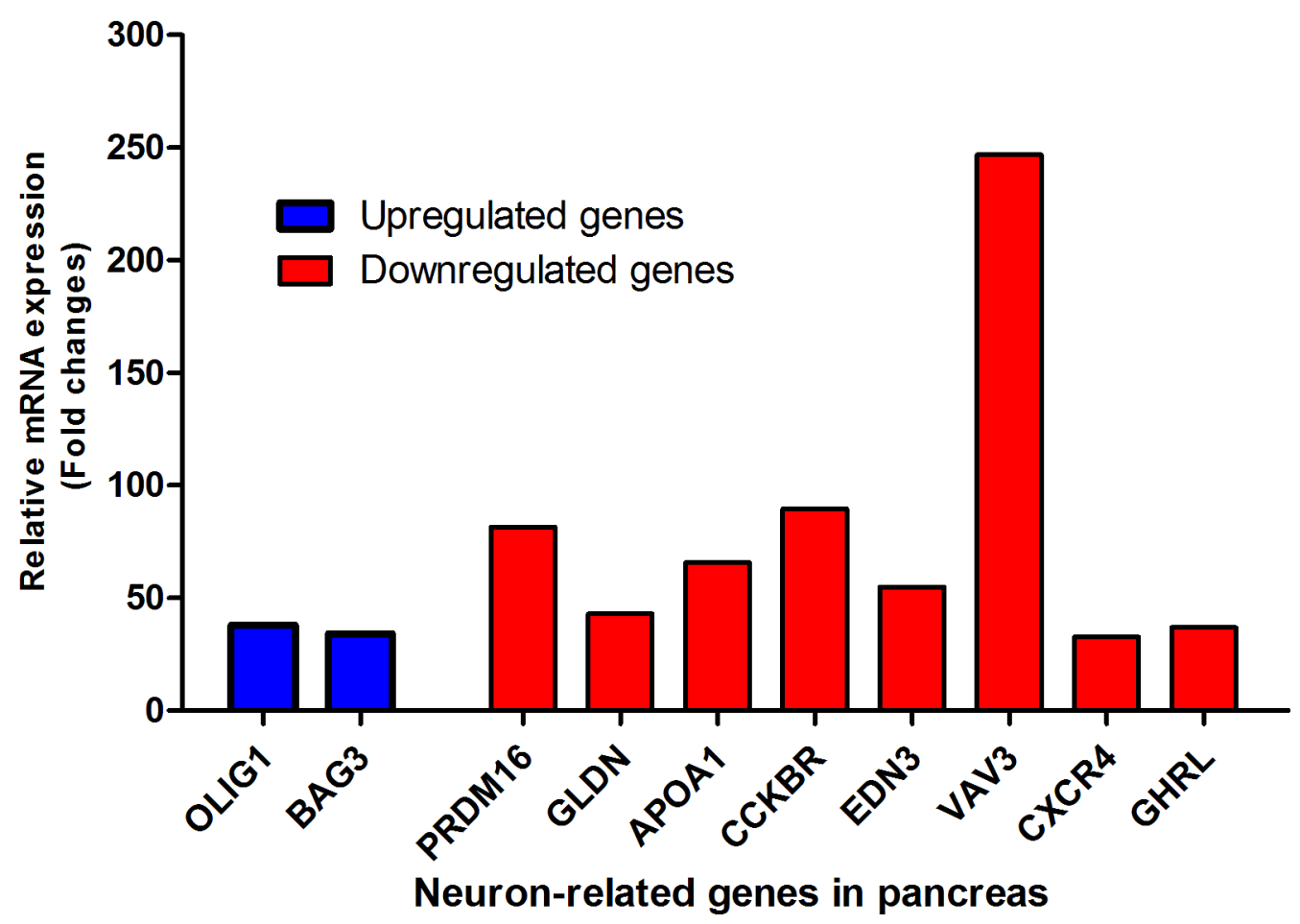

Figure 1: mRNA analysis of highly changed expression of 10 genes with a neuronal function in pancreas and pancreas with T2D. After all corrections (see M \& M), mRNA expression was expressed as ${ }^{2} \log$ values to prevent false positive gene differences between two conditions. Subsequently, Fold-change between pancreas-T2D and pancreas-control was calculated and shown on the Y-axis and was obtained from three measurements.

PPARGC1A) genes taking EGFR, SHCl and INSR part in 5 processes. Due to its high node degree [33], BAG3 resulted highly central in the whole graph. Among the others, it was connected to 8 multi-process genes, including $G 6 P D$. $A P O A 1$ was mostly connected to lipid metabolism-related genes, with 3 multi-process genes, including AKT2. SYT4 was connected to 5 genes of which only 3 were bi-process. Half of the VAV3 interactors were multi-process, while $V G F$ interacted with 7 genes, including 2 bi-process and $M A P K 3$.

\section{The ELISA of SYT4, VGF, BAG3, ApoA1, and VAV3 proteins in plasma of 41 human subjects}

To confirm the mRNA expression data derived from AT and pancreas with and without T2D, we measured five proteins SYT4, VGF, BAG3, ApoA1, and VAV3 in four different groups of 41 human subjects; 1- Lean (control), 2- Obese, 3- Obese with T2D without receiving insulin (obese+T2D-INS), and 4- Obese with T2D receiving insulin (obese+T2D+INS).

\section{SYT4 and VGF proteins}

Although the protein concentration of SYT4 was not significantly changed in obese, obese-T2D with and without receiving insulin human subjects as compared to lean group, there was a clear up-regulation trend (Figure 7A), which is in agreement with SYT4 gene up-regulation. As depicted in Figure 7B, VGF protein concentration was significantly increased in both obeseT2D with and without receiving insulin subjects as compared to lean group. Although there was no significant difference between obese and lean group, the average of VGF protein concentration was clearly higher in obese subjects than lean group (Figure 7B).

\section{BAG3, APOA1, and VAV3 proteins}

The plasma protein concentration of BAG3 was found to be similar in obese, obese-T2D without receiving insulin and lean subjects. However, there was a trend towards reduction in obese-T2D subjects receiving insulin as compared to the other three groups (Figure 8A). The protein levels of ApoA1 were not significantly reduced in all three experimental groups of patients as compared to the control lean group (Figure 8B). However, when obese-T2D subjects were injected with insulin, the plasma concentration of ApoAl goes up comparing with obese and obese-T2D without injection of insulin as displayed in Figure 8B. Plasma VAV3 protein levels (Figure 8C) show a similar pattern as ApoA1, which is 


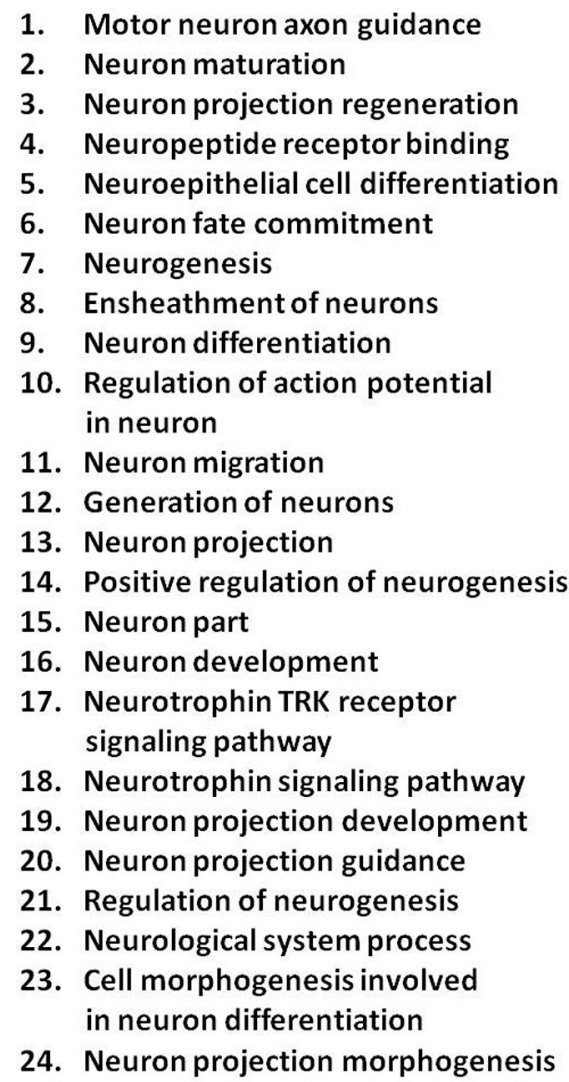

1. Motor neuron axon guidance

2. Neuron maturation

3. Neuron projection regeneration

4. Neuropeptide receptor binding

5. Neuroepithelial cell differentiation

6. Neuron fate commitment

7. Neurogenesis

8. Ensheathment of neurons

9. Neuron differentiation

in neuron

11. Neuron migration

12. Generation of neurons

13. Neuron projection

14. Positive regulation of neurogenesis

15. Neuron part

16. Neuron development

. Neurotrophin TRK receptor signaling pathway

18. Neurotrophin signaling pathway

19. Neuron projection development

20. Neuron projection guidance

Regulation of neurogenesis

22. Neurological system process

Cell morphogenesis involved

24. Neuron projection morphogenesis

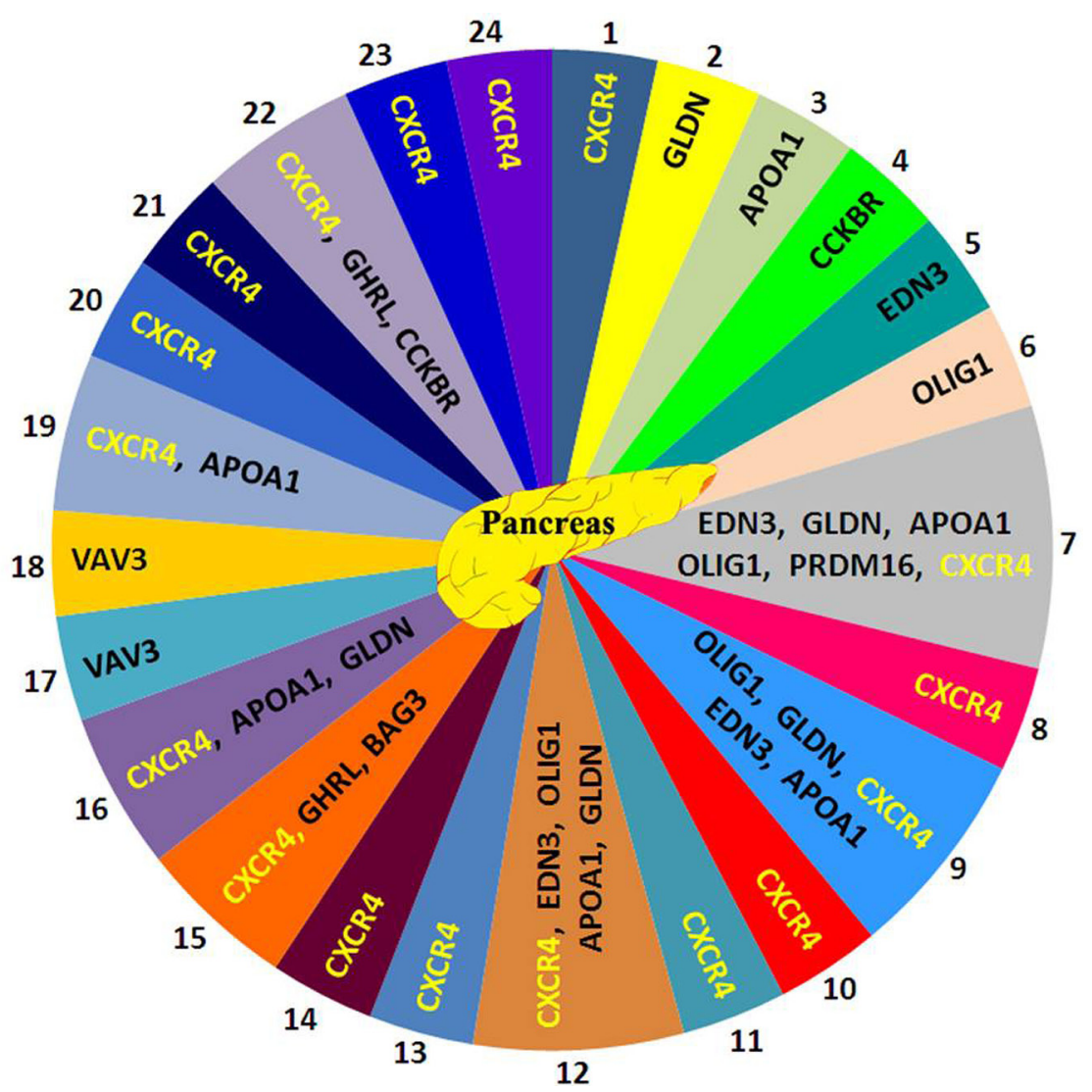

Figure 2: 24 neuronal functions related to the 10 genes found in pancreas based on Gene Ontology database search. Neuronal processes related to the 10 genes are numbered from 1 to 24 (left side of Figure) and corresponded with numbers 1 to 24 shown in pancreas Figure. Abbreviations are in Tables 1 and 2.

mirrored by down-regulation of ApoA1 and VAV3 genes. All statistical analysis obtained by ANOVA and ANCOVA was summarized in Table 3.

\section{DISCUSSION}

Aging can be expressed simply as "functionality reduction of biological process" or as "loss or reduction of the regulated cell function". Of note, the aging process can be distinguished between early or premature aging and regulated (normal) or healthy aging. Thus, all diseases such as obesity, IR, diabetes, degenerative disease, and cancer could be just referred to aging or aging-related diseases. The discovery of the factors responsible for premature aging or healthy aging will provide us information to correct premature aging or extend healthy aging [23].

Although the underlying mechanisms for the development of human T1D (autoimmune disease) are completely different from T2D, insulin signaling pathway, glucose levels, and energy storage components are involved in both types of diabetes. Both age-induced T1D and T2D are associated with many complications such as DNs. We hypothesized that AT and pancreas could be involved in the development of DNs in age-related T2D. $\mathrm{AT}$ is important during the insurgence of insulin resistance in the frame of obesity and produce factors involved in energy metabolism, such as chemokines/cytokines, and the pancreas is implicated in insulin production. There is little known about the genes involved in DNs in these two organs (with or without T2D). This study was designed to select only genes with very highly up-regulated or downregulated in AT and pancreas with age-related T2D (the cut off was adjusted to $\geq 32\left(=2^{5}\right)$ times). This selection criterion was chosen to prevent false positive gene differences between control and T2D in these two organs of human

Overall, 10 genes with a neural function were found in pancreas-T2D of which 8 genes were highly downregulated and 2 genes highly up-regulated. Although it is difficult from our studies to infer about the mechanism behind each gene in the development of DNs, we assume that these 10 genes could be potentially major players in DNs.

One of the functions of CXCR4 is known to regulate neuronal signaling and promotes hippocampal-neuron survival [34-38], while the mutation affected the gene and, in turn, suppression of $C X C R 4$ function. Thus, down-regulation 
Table 2: The list of highly up-regulated and down-regulated genes with a neuronal function detected in human control adipose tissue (AT) and AT-T2D using mRNA expression

\begin{tabular}{|c|c|c|c|c|c|}
\hline Protein ID & Protein description & pI & Mw (Da) & Gene name & $\begin{array}{l}\text { AT-T2D vs AT-CONT } \\
\text { (fold change) }\end{array}$ \\
\hline Q9H2B2 & Synaptotagmin-4 & 8.72 & 47,958 & SYT4 & 362.08 \\
\hline Q9Y2G1 & Myelin regulatory factor & 7.05 & 124,397 & $\begin{array}{l}\text { C11ORF9/ } \\
\text { MYRF }\end{array}$ & 95.06 \\
\hline Q9NQC3 & Reticulon-4 & 4.42 & 129,931 & RTN4 & 49.36 \\
\hline P18509 & Pituitary adenylate cyclase-activating & 9.83 & 18,835 & ADCYAP1 & 48.92 \\
\hline P21246 & Pleiotrophin & 9.66 & 18,942 & PTN & 46.90 \\
\hline P37088 & Amiloride-sensitive sodium channel alpha & 7.47 & 75,704 & SCNN1A & 46.59 \\
\hline Q8WUT4 & Leucine-rich repeat neuronal protein 4 & 6.82 & 78,843 & $\begin{array}{l}\text { C20ORF75/ } \\
\text { LRNN4 }\end{array}$ & 42.98 \\
\hline Q9P2J2 & Protein turtle homolog A & 6.74 & 126,58 & IGSF9 & 35.06 \\
\hline O15240 & Neurosecretory protein & 4.76 & 67,258 & VGF & -119.29 \\
\hline P24530 & Endothelin B receptor & 9.15 & 49,644 & EDNRB & -41.21 \\
\hline P47928 & DNA-binding protein inhibitor ID-4 & 8.69 & 16,622 & ID4 & -38.44 \\
\hline Q9UBY5 & Lysophosphatidic acid receptor 3 & 9.53 & 40,128 & LPAR3 & -35.62 \\
\hline O14531 & Dihydropyrimidinase-related protein 4 & 6.64 & 61,878 & DPYSL4 & -35.47 \\
\hline Q7Z5W6 & $\operatorname{laminin} \mathrm{A} 1$ & 7.02 & 60,711 & LAMA1 & -34.41 \\
\hline Q9UIU6 & Homeobox protein SIX4 & 5.45 & 82,933 & SIX4 & -34.09 \\
\hline
\end{tabular}

Protein accession number, protein description, pI (isoelectric point), the Molecular weight (Mw), gene name of that protein, and fold change (AT-T2D vs AT-Control) are presented for each gene in this Table. The Mw expressed in Da. pI resulted from whole amino acid sequence of each protein and is derived from www.expasy.org.

of $C X C R 4$ function may lead to the promotion of neuronal apoptosis. Of note, the CXCR4 was down-regulated in pancreas-T2D. Neuronal survival and regulation of neuronal signaling by CXCR4 might be reduced in T2D patients, and this may play a critical role in the development of DNs. Moreover, Based on Gene Ontology database search, 17 hits with neuronal function were found for CXCR4.

To date, apolipoprotein A-1 (APOA1) was shown to be synthesized in the liver and in the small intestine and it is a major constituent protein of plasma highdensity lipoprotein (HDL). APOA1 plays a major role in reverse cholesterol transport, as it has a high capacity to drive cholesterol efflux [39-41]. Intriguingly, APOA1 was highly expressed in control pancreas but highly down-regulated in pancreas-T2D. Several studies have shown that the mutation in APOA1 is involved in the pathogenesis of polyneuropathy [42-46]. Also, APOA1 convincingly plays a role not only in the early stage but also in late neuropathy [42-46]. These studies support our finding that $A P O A 1$ is highly down-regulated in pancreas$\mathrm{T} 2 \mathrm{D}$, and it is involved in 6 neural processes.

GHRL is also known as appetite-regulating hormone; it is highly down-regulated in pancreas-T2D and shows 2 neural functions. GHRL has been reported to protect neurons from damage and to improve neurons survival [47]. This may suggest that a down-regulation of GHRL could result in a decreased neural protection and neural survival [47].

$O L I G 1$, also known as oligodendrocyte transcription factor 1 , is very highly up-regulated in pancreas-T2D with four neural function hits and is involved in the formation of oligodendrocytes and together with $O L I G 2$ establish precursor motorneuron ( $\mathrm{pMN}$ ) of embryonic neural tube [48]. Based on literature search, we could only find one study related to up-regulation of $O L I G 1$ and disease [49]. In this study, OLIG1 was highly expressed in oligodendrogliomas and considered as biomarker for glial brain tumors. Thus, the up-regulation of OLIG1 in pancreas-T2D suggests that new studies are crucial to better elucidate $O L I G 1$ function (s).

The major action of $V A V 3$ (also guanine nucleotide exchange factor $(G E F)$ ) is in angiogenesis and it has been recently shown that loss of $V A V 3$ in mice on chow diet resulted in liver steatosis and age-induced T2D [50]. Surprisingly, when the same mice were fed a high fat diet, they showed resistance against diet-induced obesity and metabolic syndrome [51]. However, our findings 


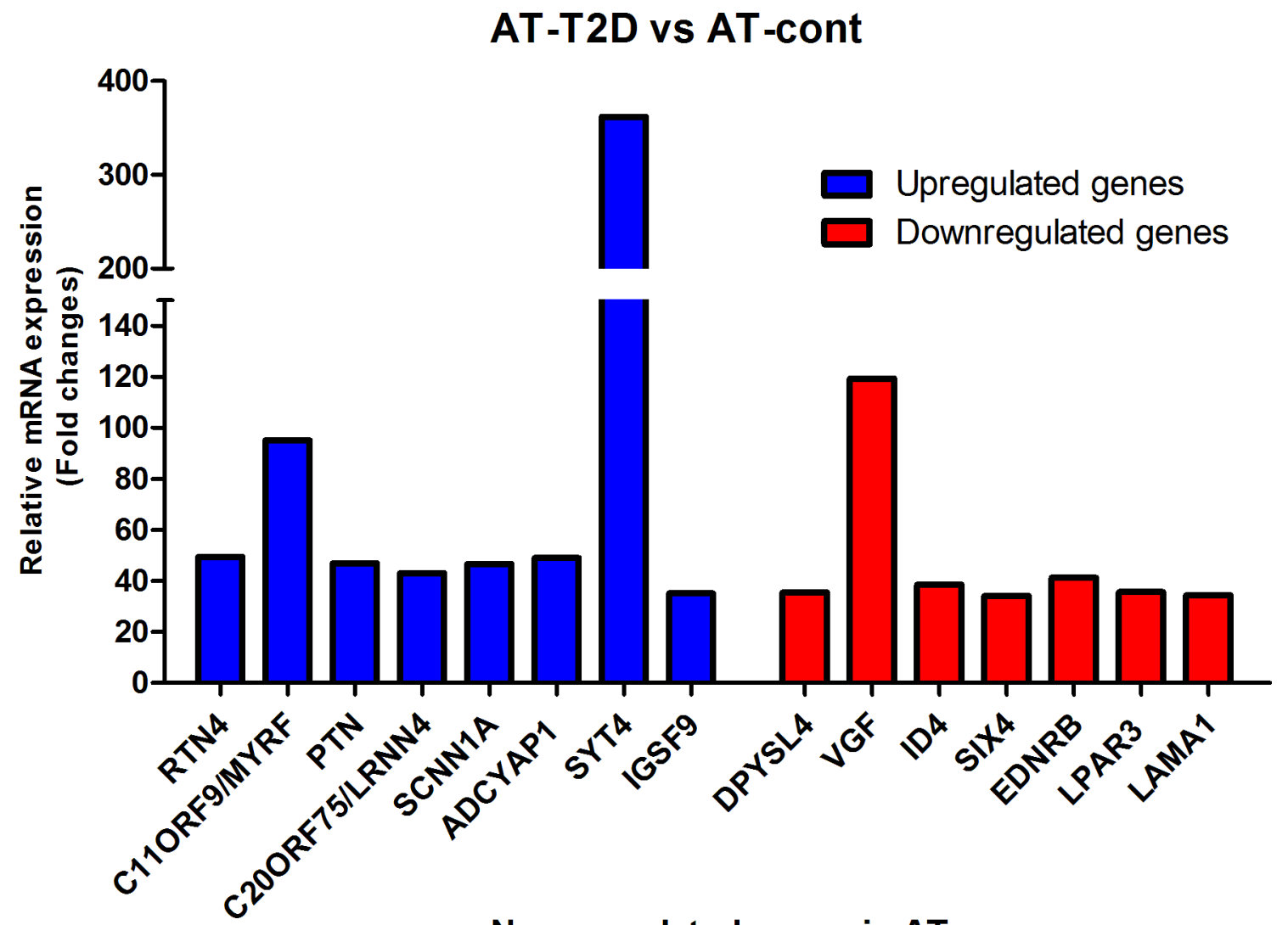

Neuron-related genes in AT

Figure 3: mRNA analysis of highly changed expression of 15 genes with a neuronal function in AT and AT with T2D. After all corrections (see M \& M), mRNA expression was expressed as ${ }^{2} \log$ values to prevent false positive gene differences between two conditions. Subsequently, Fold-change between AT-T2D and AT-control was calculated and shown on the Y-axis and was obtained from two measurements.

in humans do not support the data collected from mice deficient in VAV3. VAV3 showed a down-regulated of approximately 246 -fold in human pancreas with T2D as compared to control pancreas. This finding indeed suggests that the action of $V A V 3$ in human is completely different from that in mice with manipulated $V A V 3$ gene: the restoration of $V A V 3$ in pancreas-T2D could contribute to less severe DNs.

In AT-T2D, 8 genes were highly up-regulated and 7 genes down-regulated with neuronal hits. It is beyond the scope of this study to unravel and discuss the individual mechanism of each gene in the development of DNs. We assume that these 15 genes could be potentially major players in DNs. This study provides preliminary evidence for further investigations.

SYT4 (Synaptotagmin-4) and VGF (neurosecretory protein) genes are dramatically up-regulated and downregulated in AT-T2D, respectively. SYT4 is mainly detectable in brain and neuroendocrine system and it has been suggested to have a neuroendocrine role $[52,53]$. These authors showed that up-regulation of SYT4 blocked the release of oxytocin, which in turn resulted in an obese phenotype $[52,53]$. On the contrary, down-regulation of
SYT4 normalized oxytocin release, indicating that obesity and its afflictions are under regulation of hypothalamic neuropeptides [52, 53]. Based on our novel finding (overexpression of SYT4 in AT-T2D) and intriguing finding by Zhang et al. [52], we hypothesize that SYT4 can be used as a new biomarker for obesity and T2D and may be considered as prognostic marker for age-induced T2D, and negative regulation of SYT4 could potentially repair or reduce the degree of DNs.

The main function of $V G F$ (also called neuroendocrine regulatory peptide $(N E R P))$ is synaptogenesis and they are mainly present in neuron and neuroendocrine cells [53-55]. Body fluid homeostasis was also maintained by VGF through regulation of vasopressin release $[54,55]$. Moreover, VGF or VGF-derived peptides increase lipolysis in AT and in turn energy consumption in $V G F$ deficient mice (knock-out), which are lean [56]. However, $V G F$ was highly down-regulated in ATT2D, which does not support the results obtained from $V G F$ knock-out mice [56]. These contradictory results could be due to specific differences between mice and humans. Based on this evidence, we speculate that the $V G F$ up-regulation (e.g. by an agonist) together with 
1. Neuropeptide hormone activity

2. Negative regulation of neurogenesis

3. Positive regulation of neurological system process

4. Synaptic growth at neuromuscular junction

5. Cerebral cortex neuron differentiation

6. Ensheathment of neurons

7. Neuron maturation

8. Neuropeptide receptor binding

9. Neural precursor cell proliferation

10. Neuron recognition

11. Regulation of action potential in neuron

12. Regulation of neurological system proces

13. Neuromuscular junction development

14. Neural crest cell migration

15. Regulation of neuron differentiation

16. Neuroepithelial cell differentiation

17. Neuroblast proliferation

18. Regulation of neural precursor cell proliferation

19. Negative regulation of neuron differentiation

20. Positive regulation of neuron projection development

21. Neural crest cell development

22. Neuron projection terminus

23. Neuron projection extension

24. Neural crest cell differentiation

25. Regulation of neurogenesis

26. Neuron-neuron synaptic transmission

27. Negative regulation of neuron apoptotic process

28. Negative regulation of neuron death

29. Regulation of neuron projection development

30. Neurological system process

31. Neurotransmitter secretion

32. Neuropeptide signaling pathway

33. Neurogenesis

34. Neurotrophin TRK receptor signaling pathway

35. Neurotrophin signaling pathway

36. Neuron development

37. Neuron projection development

38. Neurotransmitter transport

39. Generation of neurons

40. Regulation of neuron apoptotic process

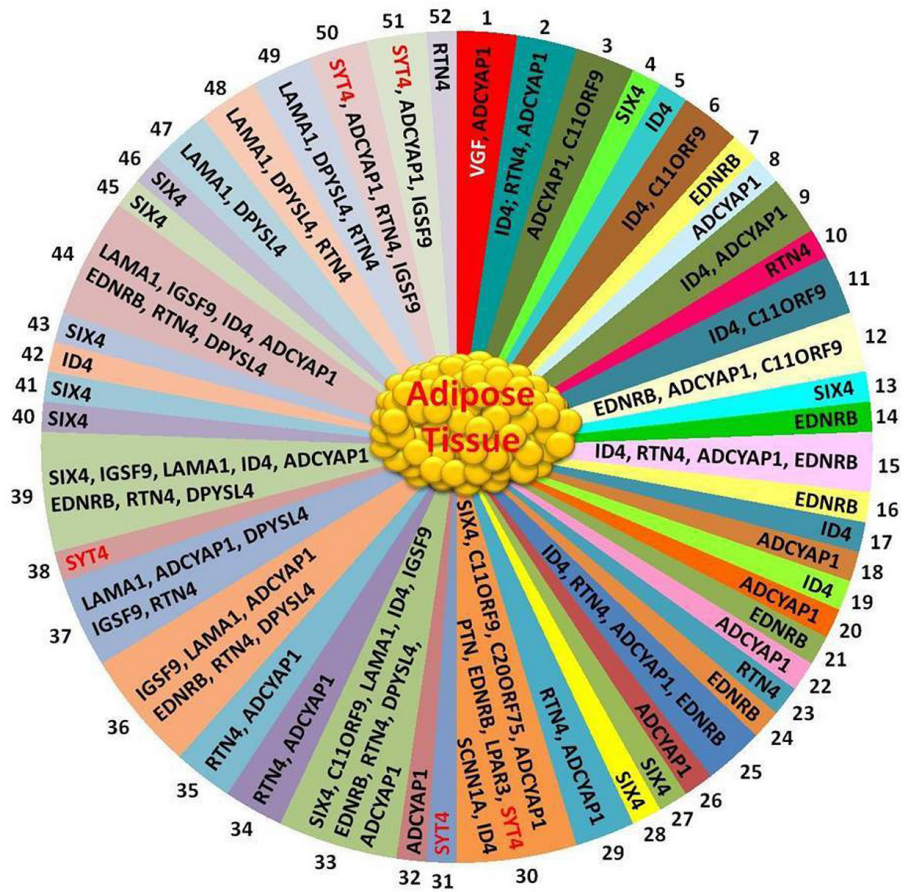

11. Regulation of neurotransmitter levels

42. Central nervous system neuron differentiation 43. Regulation of neuron death

44. Neuron differentiation

45. Neuron apoptotic process

46. Neuron death

47. Neuron projection guidance
48. Cell morphogenesis involved in neuron differentiation

49. Neuron projection morphogenesis

50. Neuron part

51. Neuron projection

52. Neuronal cell body

Figure 4: 52 neuronal functions related to the 15 genes found in AT based on Gene Ontology database search. Neuronal processes related to the 15 genes are numbered from 1 to 52 (left side and below of AT Figure) and corresponded with numbers 1 to 52 shown in AT Figure. Abbreviations are in Tables 1 and 2.
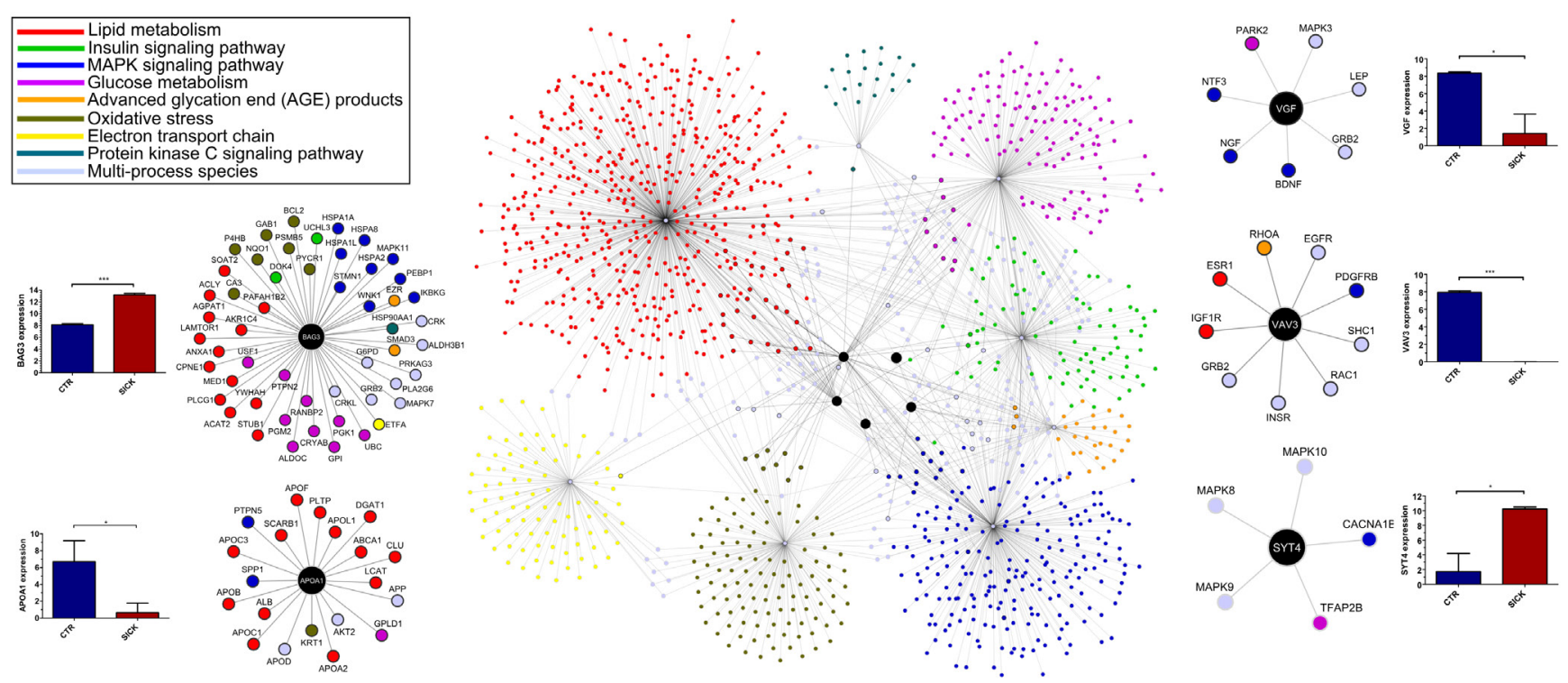

Figure 5: Functional interaction networks of the 5 most differentially expressed genes between AT and AT with T2D, and pancreas and pancreas with T2D. Reviewed interactions of SYT4, VGF, BAG3, APOA1, and VAV3 with genes participating to a list of critical biological processes and molecular functions (top-left) [source: BioGRID 3.2]. Nodes of networks are genes, while edges are interactions. Nodes are colored according to the functions/processes they participate in. Genes taking part in multiple processes are colored in cyan. Gene expression levels are represented by bars. Synaptotagmin-4 (SYT4), neurosecretory protein VGF (VGF), BAG family molecular chaperone regulator 3 (BAG3), apolipoprotein A-1 (APOA1), and guanine nucleotide exchange factor VAV3 (VAV3). 


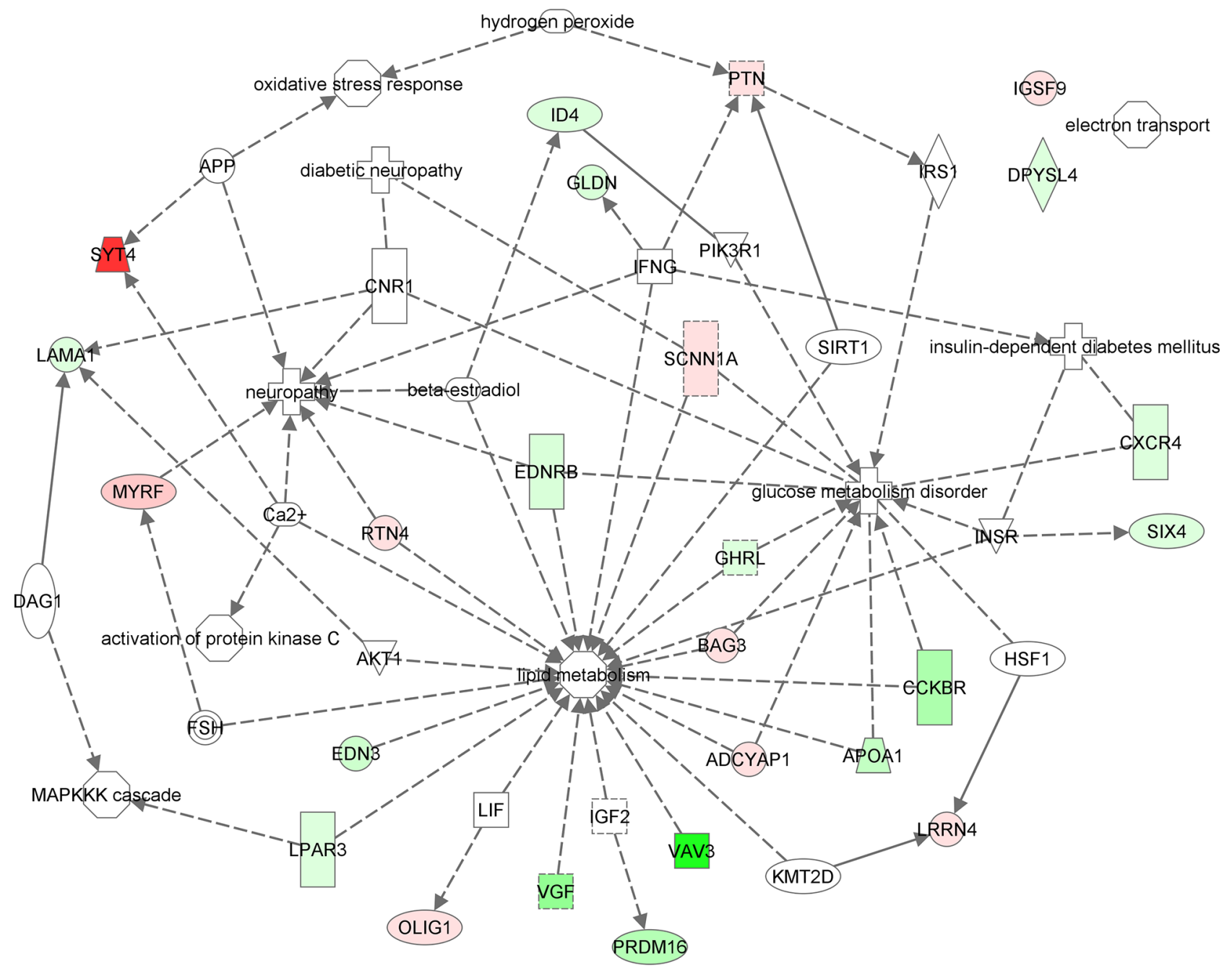

Figure 6: In-silico functional enrichment analysis of 25 differentially expressed genes between AT and AT with T2D, and pancreas and pancreas with T2D. Functional network wiring 25 differentially expressed genes between AT and AT with T2D, and pancreas and pancreas with T2D drawn by Ingenuity Pathway Analysis. Colored glyphs are genes. Octagons and crosses are relevant functions and disorders, respectively. Edges represent interactions between genes or participation of genes to functions. Dashed edges are indirect interactions.

down-regulation of SYT4 could restore the homeostatic balance between energy consumption and energy storage regulation, which could repair or reduce DNs.

Of note, the gene-expression data do not have enough power without the confirmation of gene product (i.e. protein). For this reason, five from 25 genes was selected to measure their protein levels. SYT4 and VGF proteins were chosen from AT-associated 15 genes, because SYT4 gene was highest and $V G F$ lowest expressed in AT-T2D respectively. Although plasma VGF protein concentration in three groups of patients did not correspond with $V G F$ gene expression, both plasma SYT4 and VGF protein concentrations were increased and decreased in obese-T2D with insulin injection as compared to without insulin injection in human subjects respectively. The effect of insulin was also observed for BAG3, APOA1 and VAV3 in obese-T2D with and without insulin injection in human subjects. Based on these data, it is then logic to assume that insulin has an effect on the concentrations of these five proteins and this role of insulin could be considered as so-called "corrector". Importantly, $V G F$ gene expression did not correspond to VGF protein levels and one reason could be that post translational modifications (PTMs) determine VGF protein levels and not transcriptional phase. Although we did not observe any significant differences between three patient groups and lean subjects with respect to SYT4, BAG3, APOA1, and VAV3, except for VGF protein, there was a trend between the expression of these four genes and their protein levels.

The majority of investigations and drug developments for DNs conducted so far focused on glucose metabolic pathways. Neuropathies still remains a great problem in patients with diabetes. The expression of APOA1 in control pancreas and severe down-regulation in pancreas with T2D is very novel and important finding, 

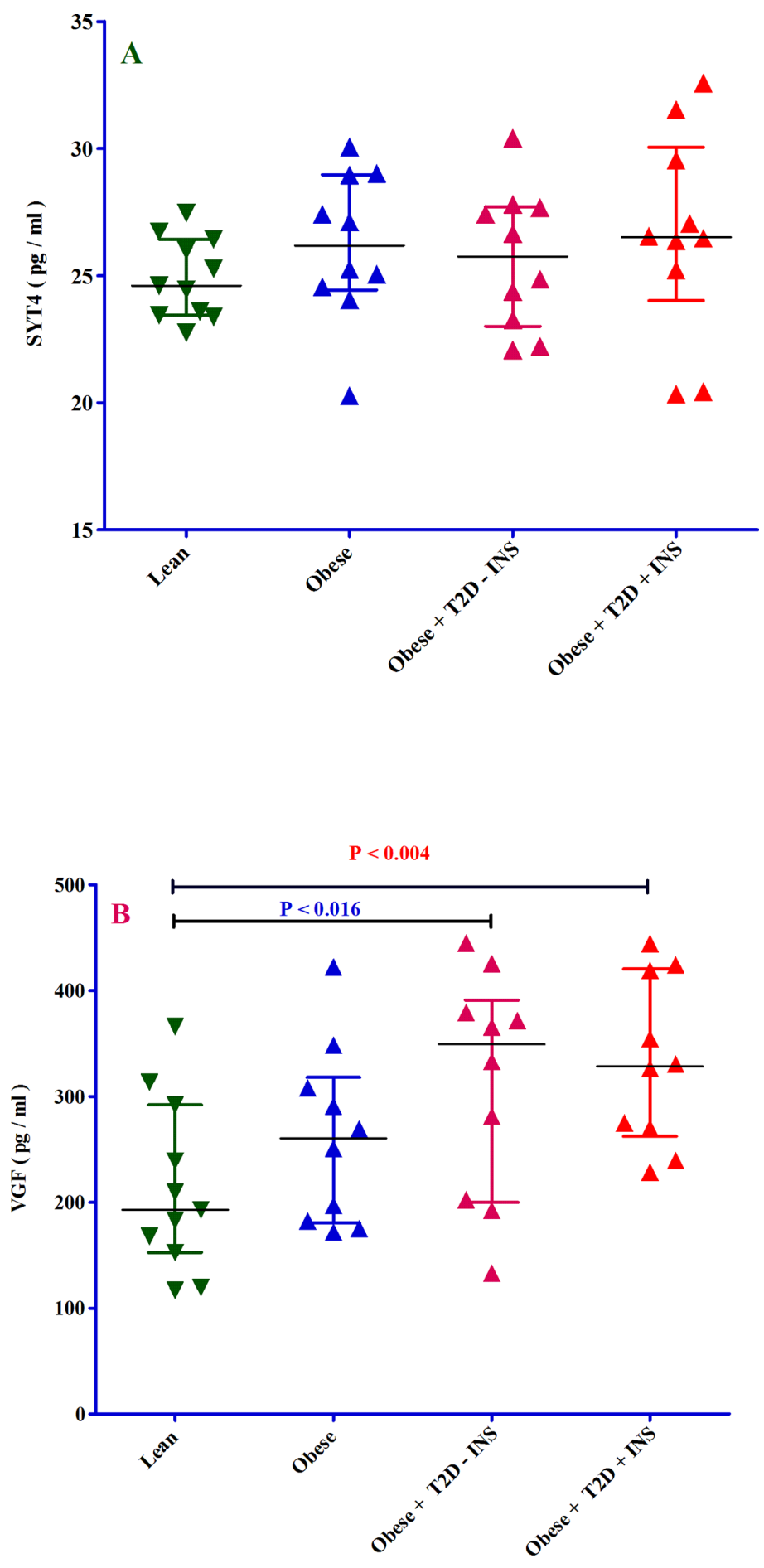

Human subjects

Figure 7: The analysis of SYT4 and VGF proteins in four different groups of 41 human subjects by ELISA. Panel A. ELISA was used to measure synaptotagmin-4 (SYT4) protein in 4 different groups of patients; 1- lean (dark green triangle), obese (dark blue triangle), 3- obese with T2D without receiving insulin (light violet triangle) and 4- obese with T2D receiving insulin (red triangle). All samples were measured in duplicate and blind. The X-axis shows the four different groups of patients. The Y-axis shows the SYT4 concentration expressed as $\mathrm{pg} / \mathrm{ml}$. Panel B. ELISA was used to measure neurosecretory protein VGF (VGF) protein in 4 different groups of patients; 1- lean (dark green triangle), obese (dark blue triangle), 3- obese with T2D without receiving insulin (light violet triangle) and 4- obese with T2D receiving insulin (red triangle). All samples were measured in duplicate and blind. The X-axis shows the four different groups of patients. The Y-axis shows the VGF concentration expressed as $\mathrm{pg} / \mathrm{ml}$. The $p$-value resulted from one-way analysis of variance (ANOVA). $P<0.05$ was accepted as statistically significant. 


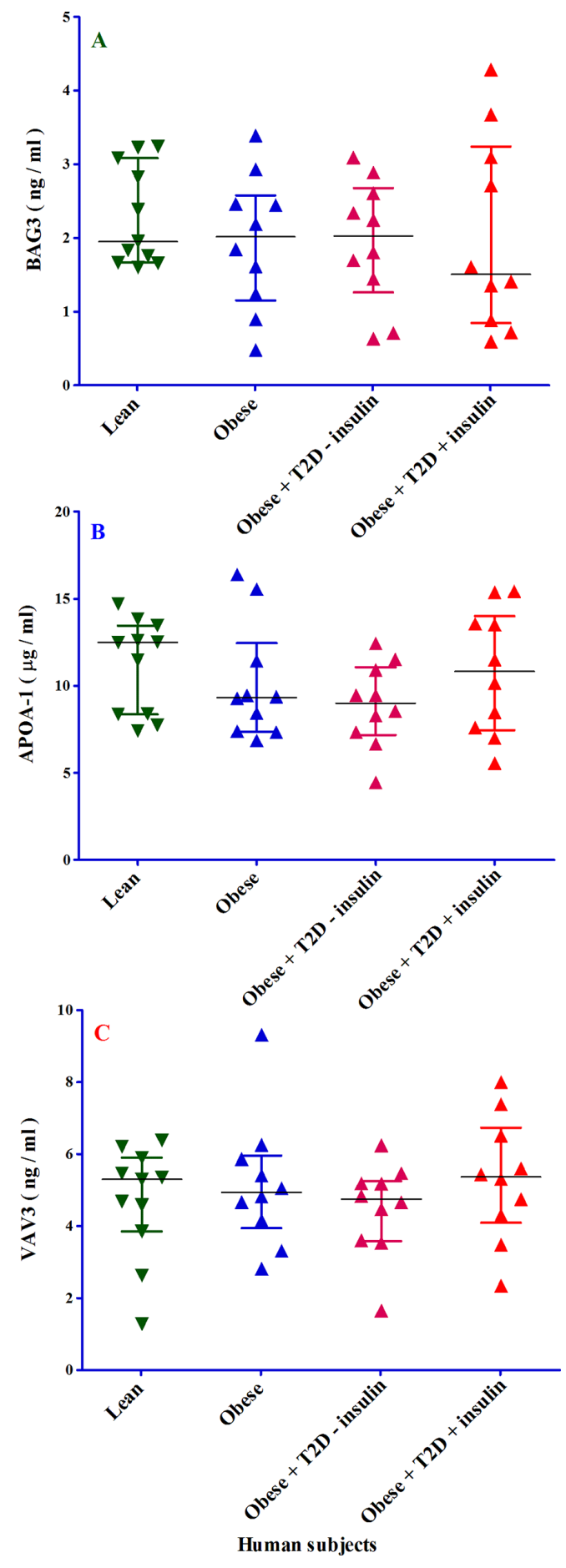

Figure 8: The analysis of BAG3, APOA1 and VAV3 proteins in four different groups of 41 human subjects by ELISA. Panel A. ELISA was used to measure BAG family molecular chaperone regulator 3 (BAG3) protein in 4 different groups of patients; 1- lean (dark green triangle), obese (dark blue triangle), 3- obese with T2D without receiving insulin (light violet triangle) and 4- obese with T2D receiving insulin (red triangle). All samples were measured in duplicate and blind. The X-axis shows the four different groups of patients. The Y-axis shows the BAG3 concentration expressed as $\mathrm{ng} / \mathrm{ml}$. Panel B. ELISA was used to measure apolipoprotein A-1 (APOA1) protein in 4 different groups of patients; 1- lean (dark green triangle), obese (dark blue triangle), 3- obese with T2D without receiving insulin (light violet triangle) and 4- obese with T2D receiving insulin (red triangle). All samples were measured in duplicate and blind. The X-axis shows the four different groups of patients. The Y-axis shows the APOA1 concentration expressed as $\mu \mathrm{g} / \mathrm{ml}$. Panel C. ELISA was used to measure guanine nucleotide exchange factor VAV3 (VAV3) protein in 4 different groups of patients; 1- lean (dark green triangle), obese (dark blue triangle), 3- obese with T2D without receiving insulin (light violet triangle) and 4- obese with T2D receiving insulin (red triangle). All samples were measured in duplicate and blind. The $\mathrm{X}$-axis shows the four different groups of patients. The Y-axis shows the VAV3 concentration expressed as ng/ml. 
Table 3: Characteristics and protein concentrations of research population groups $(n=41)$

\begin{tabular}{|c|c|c|c|c|}
\hline \multicolumn{5}{|c|}{$n(\%)$, mean $( \pm$ SD $)$} \\
\hline & $\begin{array}{c}\text { Lean }(\mathrm{BMI}<25) \\
(n=11)\end{array}$ & $\begin{array}{c}\text { Obese }(\mathrm{BMI}>30) \\
(n=10)\end{array}$ & $\begin{array}{c}\text { Obese + T2D - INS } \\
(n=10)\end{array}$ & $\begin{array}{c}\text { Obese + T2D + INS } \\
(n=10)\end{array}$ \\
\hline Gender: $n$ (Male \%) & $3(27)$ & $3(30)$ & $2(20)$ & $5(50)$ \\
\hline Age (years) & $34.09( \pm 8.08)$ & $45.20( \pm 6.23)$ & $56.26( \pm 10.54)$ & $67.18( \pm 6.97)$ \\
\hline SYT4 (pg/ml) & $24.95( \pm 1.64)$ & $26.28( \pm 3.01)$ & $25.71( \pm 2.66)$ & $26.68( \pm 4.09)$ \\
\hline VGF $(\mathrm{pg} / \mathrm{ml})$ & $212.27( \pm 80.57)$ & $261.20( \pm 85.74)$ & $309.80( \pm 105.99)$ & $330.60( \pm 78.93)$ \\
\hline VAV3 (ng/ml) & $4.75( \pm 1.64)$ & $5.14( \pm 1.81)$ & $4.53( \pm 1.31)$ & $5.25( \pm 1.69)$ \\
\hline apoA-1 $(\mu \mathrm{g} / \mathrm{ml})$ & $11.20( \pm 2.72)$ & $10.23( \pm 3.32)$ & $8.88( \pm 2.35)$ & $10.76( \pm 3.56)$ \\
\hline BAG3 (ng/ml) & $2.32( \pm 0.69)$ & $2.01( \pm 0.90)$ & $1.93( \pm 0.87)$ & $2.06( \pm 1.29)$ \\
\hline
\end{tabular}

Subjects categorized into four groups including: lean $(20.1 \leq$ BMI $\leq 25.6 \mathrm{~kg} / \mathrm{m} 2)$, obese (BMI $\geq 30 \mathrm{~kg} / \mathrm{m} 2)$, obese with T2D without receiving insulin (obese+T2D-INS) and obese subjects with T2D receiving insulin (obese+T2D+INS).

Protein concentrations were normally distributed and were presented as mean \pm SD.

which open new avenues on lipid metabolism, HDL, cardiovascular events, diabetes and its complications such as neuropathies, and will help to design new future studies. GHRL and VAV3 are also expressed in pancreas and subjected to extreme level changes in pancreas-T2D. SYT4 and $V G F$ were synthesized in AT and the levels alter in ATT2D dramatically. Based on this study, we conclude that not only diabetes but also its complications such as DNs are multifactorial diseases. Also, this study provides us a list of most important genes affected by diabetes involved in neuronal processes, which opens new avenues for new investigations for drug development (using agonists or antagonists) and new pathway(s) for the treatment of DNs.

\section{MATERIALS AND METHODS}

\section{Human biopsies and gene expression procedures}

Total RNA was isolated from human adult normal visceral AT and AT-T2D. Total RNA was collected from 5 pooled human normal pancreases and one pancreas with T2D provided by AMS Biotechnology (Amsbio, England). The quality and concentrations of RNAs were assessed in a Bioanalyzer (Experion), (Bio-Rad, USA/ The Netherlands) using the Agilent RNA 6000 Nano kit (Agilent, The Netherlands). Amplification Kit was applied to amplify and label the RNA (Applied Biosystems, The Netherlands). Total RNA was reverse-transcribed into cDNA, and the concentrations were determined in NanoDrop. RNA Amplification Kit (Ambion, USA) was used to biotinylate cRNA according to the manufacturer's instructions. Samples were purified using the RNeasy kit (Qiagen, The Netherlands). Hybridization to the Sentrix Human Expression BeadChip, washing, and scanning were performed according to the BeadStation 500 manual (revision C). One BeadChip with 12 samples was used. Each slide was scanned immediately. After the scanning, the following steps were performed: 1: quality check; 2: background correction; 3: normalization to housekeeping genes; and 4: utile normalization of the data using Beadstudio Expression module v 3.2.7. After these 4 steps, a relative mRNA intensity of ${ }^{2} \log$ was considered cut-off. Each pancreas sample was three times and AT samples two times measured. The methods were carried out in "accordance" with the approved guidelines.

\section{Human subjects and plasma collection}

41 human subjects divided into four groups of 10 (except lean group 11 subjects), independent of age and gender, as follows: Group 1-Lean subjects with a body mass index (BMI) between 20.1 and $25.6 \mathrm{~kg} / \mathrm{m} 2$, Group 2- Obese subjects with a BMI greater than or equal to $30 \mathrm{~kg} / \mathrm{m} 2$, Group 3- Obese subjects with T2D without receiving insulin (obese+T2D-INS) and a BMI greater than or equal to $30 \mathrm{~kg} / \mathrm{m} 230$ and Group 4- Obese subjects with T2D, receiving insulin (obese $+\mathrm{T} 2 \mathrm{D}+\mathrm{INS}$ ) and BMI greater than or equal to $30 \mathrm{~kg} / \mathrm{m} 2$. All patients were wellselected based on their BMI. Blood was drawn from each subject after informed consent by venipuncture into tubes containing Ethylene Diamine Tetra Acetic Acid (EDTA) as anticoagulant. Subsequently, plasma was isolated by centrifugation at $1500 \times \mathrm{g}$ for $15 \mathrm{~min}$ at $4^{\circ} \mathrm{C}$ and stored at $-80^{\circ} \mathrm{C}$ for further analysis.

\section{The measurement of protein concentrations of SYT4, VGF, BAG3, APOA1, and VAV3 by ELISA}

96-well microtitration plates (NuncSorb) were coated with primary antibodies $(100 \mu \mathrm{l}$ at $5 \mu \mathrm{g} / \mathrm{ml}$ in PBS, $\left.4^{\circ} \mathrm{C}\right)$. After four washes with PBS, the wells are saturated with BSA ( $200 \mu \mathrm{l}, 3 \%$ in PBS, at least $2 \mathrm{~h}$ at RT). After four washes with PBS, $25 \mu \mathrm{l}$ of each human 
serum sample were deposited in the wells and protein were allowed to bind to their specific mAb during $3 \mathrm{~h}$ at RT. All proteins not specifically bound were eliminated by four washes with PBS. Then the HRP-conjugated secondary antibodies were added $(100 \mu \mathrm{l}$ at $1 \mu \mathrm{g} / \mathrm{ml}$ in PBS, 45 min at RT). After four washes with PBS, $100 \mu \mathrm{l}$ of HRP substrate (TMB: 3,3',5,5'-tetramethylbenzidine; Thermo Scientific Pierce) were deposited and reaction was allowed during $15 \mathrm{~min}$ in dark (upon oxidation, TMB forms a water-soluble blue reaction product that can be measured spectrophotometrically at $650 \mathrm{~nm}$ ). Reaction was then stopped by addition of $100 \mu \mathrm{H} 2 \mathrm{SO} 42 \mathrm{M}$ (upon acidification, the reaction product becomes yellow with an absorbance peak at $450 \mathrm{~nm}$ ). Absorbance at $450 \mathrm{~nm}$ (yellow) was monitored on a plate-reader device. Primary antibodies were purchased from SIGMA (SYT4, APOA1, BAG3), from Santa Cruz Biotechnology (VAV3) and Abcam (VGF). HRP-coupled secondary antibodies (antimouse, anti-goat, anti-rabbit) were purchased from Abcam.

\section{Interaction network-based pathway analysis}

Genes belonging to lipid metabolism, insulin, protein kinase $C$, advanced glycation end (AGE) products and $M A P K$ signaling pathways, together with those participating to the electron transport chain, oxidative stress and glucose metabolism biological processes were collected [57-58]. Considering that correlation of gene expression and protein to protein interactions have been shown to cluster genes of similar function [59], we queried UniProtKB, Mentha [60], STRING [61] and BioGRID [62] in search of any evidence of connection between these genes and BAG3, APOA1, $V G F, V A V 3$ and SYT4. Interactions were represented as edges of graphs, while nodes denoted genes, as already explained in [63]. Genes were colored according to the biological processes/pathways they were participating in. Light blue nodes represented genes that took part to multiple processes and pathways. Differential expressions of BAG3, APOA1,VGF, VAV3 and SYT4 genes between normal and patient samples were evaluated by unpaired student t-test at significance level of $\alpha=5 \%$. A global view of the functional involvement of the 25 genes into the abovementioned critical processes and pathways has been further obtained by the Ingenuity Pathway Analysis workbench. A gene-function mixed network was generated, where edges corresponded to literature-confirmed scientific evidences of interaction between any two genes. Dashed edges represented indirect interactions. Green colored genes were down-regulated in our case-study, while red genes were up-regulated. Molecule shapes correspond to as many kinds of molecules, as for functions or diseases (octagons and crosses).

\section{Statistics}

ANOVA was performed as statistical analysis to identify the protein levels of SYT4, VGF, VAV3, APOA1 and BAG3 for each BMI group (1- lean, 2- obese, 3- obese+T2D-INS and 4- obese+T2D+INS).

Analysis of covariance (ANCOVA) was used to identify differentially proteins concentrations of SYT4, VGF, VAV3, apoA1, and BAG3 across the four groups as controlling for the effects of confounding variables including age and gender. ANCOVA analysis was done using change of proteins concentrations in groups as dependent variable; ordinal variable of four groups (lean, obese, obese- T2DINS, obese-T2D+INS) as a fixed effect and the effects of confounders were controlled in analysis as covariates (Table 3). The choice for a post-hoc analysis was due to having five proteins profiles across four groups. All statistical tests were two-sided and performed at a significance level of $\alpha=5 \%$. Data analyses were performed using SPSS statistical software (version 22; SPSS Inc., Chicago, USA).

\section{ACKNOWLEDGMENTS AND FUNDING}

The authors would like to thank the Erasmus Medical Center Rotterdam for their financial support of this study.

\section{Ethics statement}

This study was approved by the Medical Ethical Committee of the Erasmus Medical Center, Medical University of Rotterdam, under MEC number: 2009242. Written informed consent was obtained from all participants. The methods were carried out in "accordance" with the approved guidelines.

\section{COMPETING FINANCIAL INTERESTS}

The authors declare no competing financial interests. Authors have nothing to disclose.

\section{Author contributions}

MR and FR wrote the paper. FR conceived, designed and coordinated the study. MV edited paper, MV and VP performed ELISA. BO, FWMDR and ES provided patients and edited paper. MP edited paper. MA and FR performed statistics. FS, MD, TM, and FR prepared the figures. FR analyzed ELISA. MMM, VK, MM, MMP, FR analyzed gene data and edited manuscript.

\section{REFERENCES}

1. Aronoff SL, Berkowitz K, Shreiner B, Want L. Glucose Metabolism and Regulation: Beyond Insulin and Glucagon. Diabetes Spectr. 2004; 17:183-190.

2. Cooperberg BA, Cryer PE. Insulin reciprocally regulates glucagon secretion in humans. Diabetes. 2010; 59:2936-2940.

3. Blagosklonny MV. Validation of anti-aging drugs by treating age-related diseases Aging. (Albany, NY) 2009; $1: 281-288$. 
4. Blagosklonny MV. Common drugs and treatments for cancer and age-related diseases: revitalizing answers to NCI's provocative questions. Oncotarget. 2012; 3:1711-1724.

5. Mallone R, Roep BO. Biomarkers for immune intervention trials in type 1 diabetes. Clin Immunol. Elsevier Inc. 2013; 149:286-296.

6. Morran MP, Omenn GS, Pietropaolo M. Immunology and genetics of type 1 diabetes. Mt Sinai J Med. 2008; 75:314-327.

7. Michels AW. Targeting the trimolecular complex. Clin Immunol. Elsevier Inc. 2013; 149:339-344.

8. Coppieters KT, Harrison LC, von Herrath MG. Trials in type 1 diabetes: Antigen-specific therapies. Clin Immunol. Elsevier Inc. 2013; 149:345-355.

9. Brower $\mathrm{V}$. When the immune system goes on the attack. EMBO Rep. 2004; 5:757-760.

10. Ostenson C-G, Sandberg-Nordqvist A-C, Chen J, Hällbrink M, Rotin D, Langel U, Efendic S. Overexpression of proteintyrosine phosphatase PTP sigma is linked to impaired glucoseinduced insulin secretion in hereditary diabetic Goto-Kakizaki rats. Biochem Biophys Res Commun. 2002; 8291:945-950.

11. Sakurai H. Treatment of diabetes in experimental animals by metallocomplexes. J Pharm Soc Japan. 2008; 128:317-322.

12. Scharfmann R, Duvillie B, Stetsyuk V, Attali M, Filhoulaud G, Guillemain G. Beta-cell development: the role of intercellular signals. Diabetes Obes Metab. 2008; 10:195-200.

13. Tal MG. Type 2 diabetes: Microvascular ischemia of pancreatic islets? Med Hypotheses. 2009; 73:357-358.

14. Rezaee F, Dashty M. Role of Adipose Tissue in Metabolic System Disorders. J Diabetes Metab. 2013; 01:S13.

15. Sanjabi B, Dashty M, Özcan B, Akbarkhanzadeh V, Rahimi M, Vinciguerra M, van Rooij F, Al-Lahham S, Sheedfar F, van Kooten TG, Spek CA, Rowshani AT, van der Want J, Klaassen R, Sijbrands E, Peppelenbosch MP, Rezaee F. Lipid droplets hypertrophy: a crucial determining factor in insulin regulation by adipocytes. Sci Rep. 2015; 5:8816.

16. Meijer K, de Vries M, Al-Lahham S, Bruinenberg M, Weening D, Dijkstra M, Kloosterhuis N, van der Leij RJ, van der Want H, Kroesen B-J, Vonk R, Rezaee F. Human primary adipocytes exhibit immune cell function: adipocytes prime inflammation independent of macrophages. PLoS One. 2011; 6:e17154.

17. Purves T, Middlemas a, Agthong S, Jude EB, Boulton a J, Fernyhough P, Tomlinson DR. A role for mitogen-activated protein kinases in the etiology of diabetic neuropathy. FASEB J. 2001; 15:2508-2514.

18. Gazzaruso C, Coppola A, Montalcini T, Baffero E, Garzaniti A, Pelissero G, Collaviti S, Grugnetti A, Gallotti P, Pujia A, Solerte SB, Giustina A. Lipoprotein(a) and homocysteine as genetic risk factors for vascular and neuropathic diabetic foot in type 2 diabetes mellitus. Endocrine. 2012; 41:89-95.

19. Pande M, Hur J, Hong Y, Backus C, Hayes JM, Oh SS, Kretzler M, Feldman EL. Transcriptional profiling of diabetic neuropathy in the BKS $\mathrm{db} / \mathrm{db}$ mouse: a model of type 2 diabetes. Diabetes. 2011; 60:1981-1989.

20. Ganesh Yerra V, Negi G, Sharma SS, Kumar A. Potential therapeutic effects of the simultaneous targeting of the Nrf2 and NF- $\kappa \mathrm{B}$ pathways in diabetic neuropathy. Redox Biol. Elsevier. 2013; 1:394-397.

21. Pop-Busui R, Sima A, Stevens M. Diabetic neuropathy and oxidative stress. Diabetes Metab Res Rev. 2006; 22:257-273.

22. Hur J, Sullivan K a, Pande M, Hong Y, Sima A a F, Jagadish HV, Kretzler M, Feldman EL. The identification of gene expression profiles associated with progression of human diabetic neuropathy. Brain. 2011; 134:3222-3235.

23. Cheng X, Siow RCM, Mann GE. Novel mutations in ATPase 8, ND1 and ND5 genes associated with peripheral neuropathy of diabetes. Antioxid Redox Signal. Elsevier Ireland Ltd. 2011; 514:469-487.

24. Rezaee F. Systems Biology and Age-Induced Diseases. J Diabetes Metab. 2015; 06:516.

25. Edwards JL, Vincent AM, Cheng HT, Feldman EL. Diabetic neuropathy: mechanisms to management. Pharmacol Ther. 2008; 120:1-34.

26. Ahluwalia TS, Lindholm E, Groop LC. Common variants in CNDP1 and CNDP2, and risk of nephropathy in type 2 diabetes. Diabetologia. 2011; 54:2295-2302.

27. Dobretsov M, Romanovsky D, Stimers JR. Early diabetic neuropathy: triggers and mechanisms. World J Gastroenterol. 2007; 13:175-191.

28. Vincent A, Russell J. Oxidative stress in the pathogenesis of diabetic neuropathy. Endocr Rev. 2004; 25:612-628.

29. Figueroa-Romero C, Sadidi M, Feldman EL. Mechanisms of disease: the oxidative stress theory of diabetic neuropathy. Rev Endocr Metab Disord. 2008; 9:301-314.

30. Sima AA. New insights into the metabolic and molecular basis for diabetic neuropathy. Cell Mol Life Sci. 2003; 60:2445-2464.

31. Yasuda H, Terada M, Maeda K, Kogawa S, Sanada M, Haneda M, Kashiwagi A, Kikkawa R. Diabetic neuropathy and nerve regeneration. Prog Neurobiol. 2003; 69:229-285.

32. Lupachyk S, Watcho P, Stavniichuk R, Shevalye H, Obrosova IG. Endoplasmic reticulum stress plays a key role in the pathogenesis of diabetic peripheral neuropathy. Diabetes. 2013; 62:944-952.

33. Sabidussi G. The centrality of a graph. Psychometrika. 1966; 31:581-603.

34. Dziembowska M, Tham TN, Lau P, Vitry S, Lazarini F, Dubois-Dalcq M. A role for CXCR4 signaling in survival and migration of neural and oligodendrocyte precursors. Glia. 2005; 50:258-269.

35. Festa L, Meucci O. Effects of Opiates and HIV Proteins on Neurons: The Role of Ferritin Heavy Chain and a Potential for Synergism. Curr HIV Res. 2012; 10:453-462. 
36. Hernandez PA, Gorlin RJ, Lukens JN, Taniuchi S, Bohinjec J, Francois F, Klotman ME, Diaz GA. Mutations in the chemokine receptor gene CXCR4 are associated with WHIM syndrome, a combined immunodeficiency disease. Nat Genet. 2003; 34:70-74.

37. Chowdhury SKR, Smith DR, Fernyhough P. The role of aberrant mitochondrial bioenergetics in diabetic neuropathy. Neurobiol Dis. Elsevier Inc. 2013; 51:56-65.

38. Liu C, Weng Y, Yuan T, Zhang H, Bai H, Li B, Yang D, Zhang R, He F, Yan S, Zhan X, Shi Q. CXCL12/CXCR4 signal axis plays an important role in mediating bone morphogenetic protein 9-induced osteogenic differentiation of mesenchymal stem cells. Int J Med Sci. 2013; 10:1181-1192.

39. Pankhurst G, Wang XL, Wilcken DE, Baernthaler G, Panzenböck U, Raftery M, Stocker R. Characterization of specifically oxidized apolipoproteins in mildly oxidized high density lipoprotein. J Lipid Res. 2003; 44:349-355.

40. Levels JH, Bleijlevens B, Rezaee F, Aerts JM, Meijers JC. SELDI-TOF mass spectrometry of High-Density Lipoprotein. Proteome Sci. 2007; 5:15.

41. Rezaee F, Casetta B, Levels JHM, Speijer D, Meijers JCM. Proteomic analysis of high-density lipoprotein. Proteomics. 2006; 6:721-730.

42. Nakata K, Kobayashi K, Yanagi H, Shimakura Y, Tsuchiya S, Arinami T, Hamaguchi H. Autosomal dominant hypoalphalipoproteinemia due to a completely defective apolipoprotein A-I gene. Biochemical and biophysical research communications. 1993; 196:950-955.

43. Ng DS, Leiter LA, Vezina C, Connelly PW, Hegele RA. Apolipoprotein A-I Q[-2]X causing isolated apolipoprotein A-I deficiency in a family with analphalipoproteinemia. The Journal of clinical investigation. 1994; 93:223-229.

44. Schmitz G, Assmann G, Rall SC, Mahley RW. Tangier disease: defective recombination of a specific Tangier apolipoprotein A-I isoform (pro-apo A-i) with high density lipoproteins. Proc Natl Acad Sci U S A. 1983; 80:6081-6085.

45. Nichols WC, Gregg RE, Brewer HB, Benson MD. A mutation in apolipoprotein A-I in the Iowa type of familial amyloidotic polyneuropathy. Genomics. 1990; 8:318-323.

46. Takada Y, Sasaki J, Ogata S, Nakanishi T, Ikehara Y, Arakawa $\mathrm{K}$. Isolation and characterization of human apolipoprotein A-I Fukuoka (110 Glu-Lys). A novel apolipoprotein variant. Biochimica et biophysica acta. 1990; 1043:169-176.

47. Spencer S, Miller A, Andrews Z. The Role of Ghrelin in Neuroprotection after Ischemic Brain Injury. Brain Sci. 2013; 3:344-359.

48. Zhou Q, Wang S, Anderson DJ. Identification of a novel family of oligodendrocyte lineage-specific basic helix-loophelix transcription factors. Neuron. 2000; 25:331-343.

49. Lu QR, Park JK, Noll E, Chan JA, Alberta J, Yuk D, Alzamora MG, Louis DN, Stiles CD, Rowitch DH, Black PM. Oligodendrocyte lineage genes (OLIG) as molecular markers for human glial brain tumors. Proc Natl Acad Sci U S A. 2001; 98:10851-10856.
50. Hunter SG, Zhuang G, Brantley-Sieders D, Swat W, Cowan $\mathrm{CW}$, Chen J. Essential role of Vav family guanine nucleotide exchange factors in EphA receptor-mediated angiogenesis. Mol Cell Biol. 2006; 26:4830-4842.

51. Menacho-Márquez M, Nogueiras R, Fabbiano S, Sauzeau V, Al-Massadi O, Diéguez C, Bustelo XR. Chronic sympathoexcitation through loss of vav3, a rac1 activator, results in divergent effects on metabolic syndrome and obesity depending on diet. Cell Metab. 2013; 18:199-211.

52. Zhang G, Bai H, Zhang H, Dean C, Wu Q, Li J, Guariglia S, Meng Q, Cai D. Neuropeptide Exocytosis Involving Synaptotagmin-4 and Oxytocin in Hypothalamic Programming of Body Weight and Energy Balance. Neuron. 2011; 69:523-535.

53. Tong Q. Synaptotagmin 4: A New Antiobesity Target? Neuron. 2011; 69:401-403.

54. Sasaki K, Osaki T, Minamino N. Large-scale identification of endogenous secretory peptides using electron transfer dissociation mass spectrometry. Mol Cell Proteomics. 2013; 12:700-709.

55. Toshinai K, Nakazato $M$. Neuroendocrine regulatory peptide-1 and -2: novel bioactive peptides processed from VGF. Cell Mol Life Sci. 2009; 66:1939-1945.

56. Fargali S, Scherer T, Shin AC, Sadahiro M, Buettner C, Salton SR. Germline ablation of VGF increases lipolysis in white adipose tissue. Journal of Endocrinology. 2012; 215:313-322.

57. The UniProt Consortium. Activities at the Universal Protein Resource (UniProt). Nucleic Acids Res. 2014; 42:D191-198.

58. Kinsella RJ, Kähäri A, Haider S, Zamora J, Proctor G, Spudich G, Almeida-King J, Staines D, Derwent P, Kerhornou A, Kersey P, Flicek P. Ensembl BioMarts: a hub for data retrieval across taxonomic space. Database (Oxford). 2011; 2011:bar030.

59. Eisen MB, Spellman PT, Brown PO, Botstein D. Cluster analysis and display of genome-wide expression patterns. Proc Natl Acad Sci. 1998; 95:14863-14868.

60. Calderone A, Castagnoli L, Cesareni G. mentha: a resource for browsing integrated protein-interaction networks. Nat Methods. 2013; 10:690-691.

61. Mering Cv, Huynen M, Jaeggi D, Schmidt S, Bork P, Snel B. STRING: a database of predicted functional associations between proteins. Nucleic Acids Res. 2003; 31:258-261.

62. Chatr-Aryamontri A, Breitkreutz B-J, Heinicke S, Boucher L, Winter A, Stark C, Nixon J, Ramage L, Kolas N, O’Donnell L, Reguly T, Breitkreutz A, Sellam A, Chen D, Chang C, Rust J, Livstone M, Oughtred R, Dolinski K, Tyers M. The BioGRID interaction database: 2013 update. Nucleic Acids Res. 2013; 41:D816-823.

63. Pazienza V, Borghesan M, Mazza T, Sheedfar F, Panebianco C, Williams R, Mazzoccoli G, Andriulli A, Nakanishi T, Vinciguerra M. SIRT1-metabolite binding histone macroH2A1.1 protects hepatocytes against lipid accumulation. Aging (Albany NY). 2014; 6:35-47. 